\title{
Graph Based Topological Analysis of Tessellated Surfaces
}

\author{
Tula Ram Ban \\ Delmia Solutions Pvt. Ltd, \\ Bangalore-560 078 (INDIA) \\ TularamBAN@delmia.com
}

\author{
Dibakar Sen \\ Department of Mechanical Engineering \\ Indian Institute of Science, \\ Bangalore-560012 (INDIA) \\ dibakar@mecheng.iisc.ernet.in
}

\begin{abstract}
In this paper a graph-based method is presented which not only characterizes topological classification of the tessellated surfaces but also simultaneously generates the substantial circles or generators on the surface. Canonical polygons cannot always be mapped back to the original surface in terms of the edges of the given triangles. Hence, instead of applying canonical transformation to the initial "word", an associated graph is constructed using the unique vertices in the word. The graph is then decomposed into its constituent loops and paths. Based on the type of edges present, the loops are classified into three types. The number of loops of each type in the graph is then used for counting the rank or genus and classification of the given surface as being open or closed, orientable or non-orientable. The image of the loops and paths on the original surface give the substantial circles and arcs on the surface respectively.
\end{abstract}

\section{Categories and Subject Descriptors}

I.3.5 [Computer Graphics]: Computational Geometry and Object Modeling - geometric algorithms, languages and systems. G.2.2 [Discrete Mathematics]: Graph Theory - graph labeling, path and circuit problems.

\section{General Terms}

Algorithms.

\section{Keywords}

Computational topology, simplical complex, surface classification, characterization, cut lines.

\section{INTRODUCTION}

A tessellated surface as a representation is simple and versatile in the sense that it can represent surfaces of arbitrary topology. However, the topological nature of the surface is generally implicit which calls for its topological verification for modeling or topology identification for surface parameterization as in reverse engineering $[1,10,13,16,17,26,29,33]$. Topology identification makes geometric computations more efficient and meaningful $[6,7,27]$.

Characterization of manifold surfaces involves identification of the topological invariants associated with a class of surface. This has been carried out in literature by using sphere-handle convention [5], Betti numbers and Euler characteristics [12,28],

Permission to make digital or hard copies of all or part of this work for personal or classroom use is granted without fee provided that copies are not made or distributed for profit or commercial advantage and that copies bear this notice and the full citation on the first page. To copy otherwise, or republish, to post on servers or to redistribute to lists, requires prior specific permission and/or a fee.

SM'03, June 16-20, 2003, Seattle, Washington, USA.

Copyright 2003 ACM 1-58113-706-0/03/0006...\$5.00.
Morse theory, Reeb graph and polygon schema [14], substantial arc/circles [5] etc. Euler characteristic relates the geometric features (vertex, edge, face) of a closed orientable surface with its global topological nature (genus, components etc.) and is applicable mostly for polyhedral solids. Morse theory relates local differential geometric attributes of a closed smooth surface and its global topological nature. Canonical polygon based characterization in some sense is more general in that it characterizes both orientable and non-orientable closed 2manifolds [14]. Substantial arc/circles based characterization of surfaces [5] considers the set of non-homoeomorphic curves/surfaces on the surface whose counts are used to identify the invariants associated with a class of surface. This method is suitable for characterizing even open surfaces. Application of topology in geometric modeling has been mostly done for validating a model using Euler characteristics [12,28]. Computational topology based modeling schemes have been suggested in $[2,11,19,20,21,32]$. Utility of topological information in different fields including robotics, computer aided drug design, genetics, chemical synthesis, flow visualization etc. has been indicated in [3,6,25]. Hilga et al. [22] employed a Reeb graph based approach to 3D shape recognition problems.

Extraction of topological features of geometric models is computationally challenging. Vegter and Yap [34] presented an $\mathrm{O}(n \log n)$ algorithm for computation of canonical polygonal schema of a tessellated surface, where $n$ is the total number of vertices, edges and faces on the surface. Kartasheva [23] considered a cell complex in 3D consisting of tetrahedrons which makes it applicable only for solids (oriented, closed boundary surface). Also, the surface based algorithms also can be extended to determine the cutting sections that, in any case, cannot be guaranteed to be planar. Lazarus et al. [24] gave a $\mathrm{O}(g n)$ algorithm for the closed orientable surfaces, where $g$ is the genus of the surface. The method marches through all the triangles in the surface for construction of a spanning tree. The map of the generators on the surface is very complex and non-intuitive. A still improved $\mathrm{O}(n)$ algorithm for construction of non-canonical but optimal sized polygonal schema and $\mathrm{O}(n+k \log g)$ algorithm for detection null-homotopy cycles of size $k$ is proposed in [8]. Apart from that one can find work concerning extraction and application of topological information based on Morse theory [4, 31], knot theory [15] etc. Application of topological dissection to homeomorphically deforming (flattening) the surface is reported in $[10,33]$ which could in turn be used for global parameterization of surfaces in reverse engineering. In $[9,30]$ it is theoretically proved that the problem of topological equivalence could be reduced to a problem of graph isomorphism for general (nonmanifold) 2-complexes. However, such objects are sparingly encountered in practice; graph isomorphism test in itself is difficult and the necessity to consider all the triangles for constructing the graph is likely to make algorithms resource 
intensive. There is a need for simpler methods for topological analysis of surfaces for their wider applicability.

Although Euler characteristic, Betti numbers and Morse numbers all characterize compact 2-manifolds, it is the polygonal schema and substantial arc based characterizations which can actually construct the homeomorphism as a step towards global parameterization, texture mapping etc. Even though substantial arcs/circles provide a geometrically simple, direct and more general way of classifying surfaces, computationally identifying the best of these characteristic curves (minimum cut [10]) on the surface is difficult (NP-hard). On the other hand, although canonical words under polygonal schema give a simple method for classifying closed 2-manifolds and generation of a word polygon is conceptually straight forward, reducing it to canonical form is difficult because it involves a great deal of pattern search and symbolic manipulation of the word. Even more difficult is the process of mapping the canonical polygon on the given surface. In this paper we propose to unify the two concepts in a single characterization by using a graphs associated with any word [14], a non-canonical polygon, for identification of generators on the surface. Edges in the graph are coloured. The number of circuits with different kinds of edges in the graph completely characterizes the given surface because their image on the given surface correspond to the substantial or boundary circles; the paths not participating in any circuit correspond to the substantial arcs on an open surface.

\section{CONVENTIONAL SURFACE CLASSIFICATION SCHEMES}

Topological classification of surfaces requires identification of homeomorphism among surfaces. Two surfaces are considered topologically equivalent (homeomorphic) if there is a continuous transformation that is continuously invertible [5]. As given in [14], any smooth, compact, connected surface (without boundary) is homeomorphic (and diffeomorphic) either to a sphere with $g$ handles, where $g \geq 0$, or to a sphere with $k$ cross-caps, where $k>0$; a surface of the first type cannot be homeomorphic to one of the second type, nor can surfaces with different values of $g$ or $k$ be homeomorphic. Also, since a handle on a non-orientable surface is equivalent to two cross-caps [14], a sphere with $x$ handles and $y>0$ cross-caps, is homeomorphic to a sphere with $2 x+y$ crosscaps.

\subsection{Substantial Arcs and Circles for Surface Characterization}

A substantial arc is a cut line that does not separate the surface into unconnected domains. On a closed surface, the corresponding entity is called a substantial circle [5]. The maximum number of substantial arcs/circles that can be simultaneously present on a surface, without separating it, is called the rank of the surface. Two surfaces are homeomorphic if and only if they have the same rank, same number of boundary circles and are either both orientable or both non-orientable. When a surface is cut along the maximum number of substantial arcs/circles, it becomes equivalent to a disc. The bounding circle of this disc contains pairs of segments (mates), which are parts of the substantial arcs/circles. As one traverses the edges of the disc (indicated with an arrow in Fig.1(b)), if the end vertices of an edge and its mate is found to occur in the same order, the surface is non-orientable, otherwise it is orientable. Rank of closed orientable surfaces is even; half rank is called the genus of the surface, which is same as the number of handles on a sphere.

\subsection{Canonical Polygon Schema for Closed Surface Characterization}

Every smooth compact 2-manifold (surface) can be triangulated with finitely many triangles. Providing a sense to these triangles and cutting the surface along these edges we get a set of triangles with oriented edges. Two triangles with a matching edge can be glued together to get a polygon (quadrilateral); other triangles in the set can be glued to the polygon in a recursive fashion by identifying one of the edges of a triangle with a boundary edge of the polygon. Marking the edges of the polygon with a label the polygon can be coded as a "word". Using a sequence of symbolic transformations involving grouping, reordering, renaming and removal of edges $[13,34]$ any word can be reduced to one of the following three canonical forms.

$$
\begin{aligned}
& \text { (1) } W=a a^{-1} \\
& \text { (2) } W=a_{1} b_{1} a_{1}{ }^{-1} b_{1}^{-1} \ldots a_{g} b_{g} a_{g}^{-1} b_{g}^{-1}, \\
& \text { (3) } W=c_{1} c_{1} c_{2} c_{2} \ldots c_{k} c_{k} \\
& \text { where } g \text { and } k \text { are positive numbers. }
\end{aligned}
$$

Here, (1) corresponds to a sphere, (2) corresponds to a sphere with $g$ handles and (3) corresponds to a sphere with $k$ cross-caps. A word expressed in its canonical form is also referred to as canonical polygon in this paper.

\subsection{Relationship Between Substantial Circles and Word Polygons}

The canonical polygon corresponding to the canonical word consists of even number of edges with duplication of edge labels. These labels either occur adjacent to each other, when their traversal in the polygon is in the same sense, or alternate with another edge, when their traversal in the polygon is in the opposite sense. When these edges are glued together respecting the sense, each mated edge in the word manifest as a substantial circle on the surface with the specialty that all substantial circles are mapped distinctly on the surface but for one point, which is shared by all of them. However, a general set of substantial circles on a given surface has a wide variety of topological relationship, as discussed in section-3.2 below. On the other hand, dealing with a given tessellated surface, there may not be any vertex with enough number of edges incident on them, or such a vertex may not belong to the canonical polygon. A triangulated double torus requires at least one vertex with degree eight which may not be available (Fig.4(a)). This implies that computationally mapping the canonical polygon on the surface using the edges of the given tessellation is difficult and sometimes impossible.

\section{GRAPH BASED CHARACTERIZATION}

In the following a method is described which, by using a graph of the word, obtains the substantial arcs and circles in terms of the original edges of the triangles representing the surface $S$, from a word in its non-canonical form and classifies the surface using the features of the graph. The graph simplification algorithm minimizes the lengths of the cut lines in terms of the number of edges in them. The methods use only the connectivity information of the constituent triangles. 


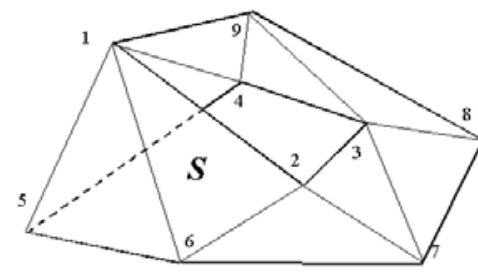

(a) Möbius Strip Surface

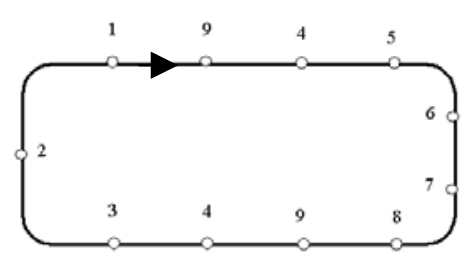

(b) Word Polygon of Mōbius Strip
Triangles:

156126145149267237378389349

Polygon Steps (common edge underlined): $15 \underline{61}+12 \underline{161}=15621$

$15621+1451=145621$

$\underline{14} 5621+\underline{1491}=1945621$

$1945 \underline{621}+\underline{2} 672=1945672$

$19456721+2372=19456732$

$194567321+3783=194567832$ $1945678321+3893=19456789321$ $19456789321+3493=19456789432$ Equivalent Word $=a b c$ defgohi

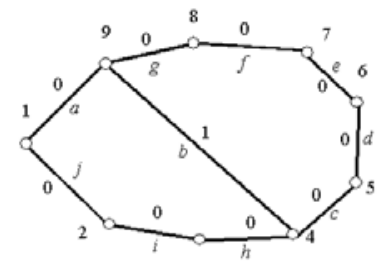

(c) Graph of Möbius Strip

Figure 1: Graph construction for an open non-orientable surface, Möbius strip

\subsection{Word and Associated Graph}

Word Polygon: To construct the word, first the polygon is constructed as a sequence of vertices. Edges are identified using two consecutive vertices in a triangle or a polygon. We start from any triangle as the starting polygon and glue an edge of an adjacent triangle to it to get the updated polygon, which in effect adds a vertex in the polygon. At each step we eliminate redundant edges as identified by occurrence of the vertex pattern, ...x,y, x... in the polygon adjacent the newly added vertex to maintain the polygon of minimum length. When all the triangles have been added, we have the required polygon (Fig.1(b))

Edge Classification: As we traverse the polygon (indicated with an arrow in Fig.1(b)), the first occurrence of an edge is considered positive. Subsequent occurrence of the edge is considered positive if the delimiting vertices appear in the same order, negative otherwise.

Graph of a Word: For a word polygon $P$, the graph, $G(V, E)$, is defined as follows: the set of unique vertices in $P$ gives $V$ and $E$ is constructed by using pairs of vertices that are adjacent in $P$. Let, $W$ be the word represented as the sequence of edges of $P$ with appropriate classification. Thus $G$ is completely defined by keeping only the unique edges in $W$. The edges in $G$ are coloured by using a tag indicating the nature of its occurrence in $W$. This we call the order of the edge. Since we are not assuming the given surface to be a closed manifold, in $W$ an edge may occur once or twice where the second occurrence may have either positive or negative classification. If an edge appears only once in $W$, its order is 0 ; if an edge appears twice with opposite sense, its order is -1 and if an edge appears twice with same sense, its order is 1. Fig.1(c) illustrates the graph construction for a triangulated Möbius strip.

\subsection{Graph and the Characteristic Curves on a Surface}

Mapping: There are three types of characteristic curves on a surface: substantial arcs, substantial circles and boundary circles. Since the graph has been created from the word without bringing it to the canonical form, the vertices and edges in the graph, $G$, can be easily identified on the original surface, $S$. It is easy to see in Fig. 2 that homologous substantial circles on $S$, can be

identified with the circuits in $G$. In the case of open surfaces (Fig.2(h),(i)), the boundary circles also get mapped to the circuits of $G$ and the homologous substantial arcs can be mapped to the cut edges [18] of $G$ (Fig.2(i)) or to paths not participating any of the circuits identified (Fig.1(c)).

Relationship Among Characteristic Curves: When the maximum number of substantial circles/arcs or cut-lines are introduced on a surface, the surface is homologous to a disk, which in turn is homologous to the canonical polygon. However, there are many different ways the cut-lines can be introduced, each with a probably different topological relationship among the arcs of the circles. Fig. 2 shows some of the different ways the cut-lines can be introduced on a torus and a double-torus along with the graph of the cut-lines. It can be observed that, even though the graph has different number of vertices and edges (hence non-isomorphic), the number of independent cycles in the graph is same for the homologous objects and is equal to the

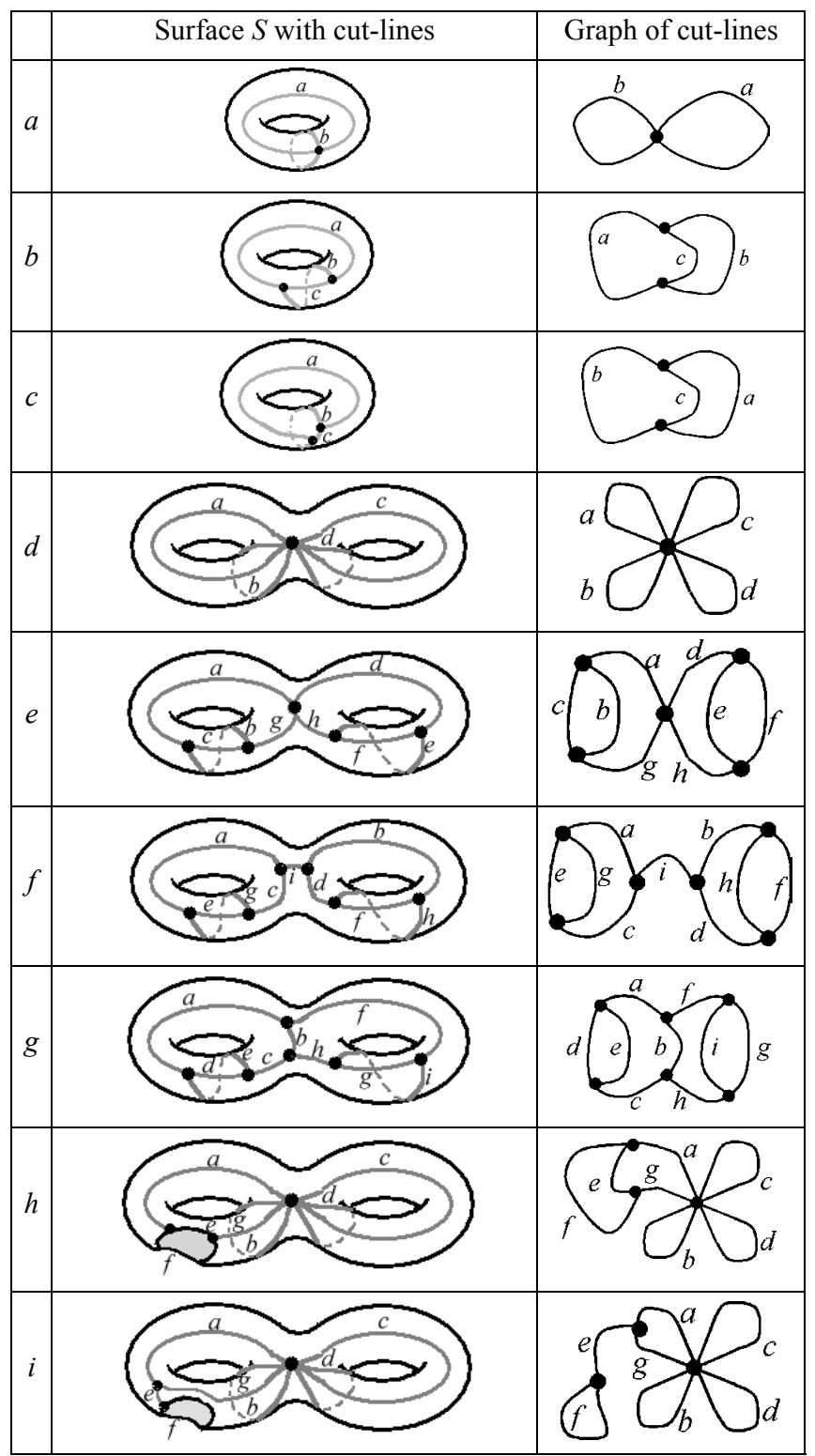

Figure 2: Relationship among characteristic curves 
number of substantial circles the surface can accommodate. When the surface has boundary, the boundary circles would also appear as cycles in the graph. Thus the type of cycle/circles is an independent information.

Graph Simplification: The purpose of graph simplification is to reduce the lengths of the circuits so as to get the shortest characteristic curves on the surface. The idea is, if in a circuit, two consecutive edges belong to the same triangle, the graph can be modified by introducing the third edge of the triangle and modifying the previous graph. As shown in Fig.3, the modification can be performed in two ways: by vertex deletion and edge swap. If the degree of the vertex common to the two earlier edges is two, the vertex and the two earlier edges can be deleted. In the second case, where the degree of the vertex common to the two earlier edges is more than two, the vertex cannot be deleted but one of the earlier edges can be deleted. This is called edge swap and it is performed if the operation increases the degree of some vertex or it facilitates a vertex deletion in the next step. The graph, $G$, in Fig.4(c) is simplified to the graph, $G^{*}$, in Fig. 4(d) with shortest substantial circles (in terms of number of edges rather than their geometric length) in the present case.

Classification of Fundamental Circuits: Since edges of $S$ are shared at most twice, $G$ is always planar [18] even though $S$ is topologically non-planar [4]. Hence the number of fundamental circuits, in $G$ is $e-v+1$, where $v$ and $e$ are the number of vertices and edges in $G$. These fundamental circuits are classified as follows: order 0 circuits are circuits with all edges of order 0 ; an order -1 circuit does not have order 1 edges but it has at least one order -1 edge; an order 1 circuit has at least one order 1 edge.

Extraction of Fundamental Circuits: The fundamental circuits of $G$ can be extracted by constructing the minimum spanning tree

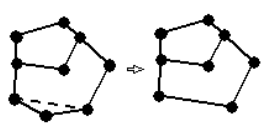

(a) Vertex deletion

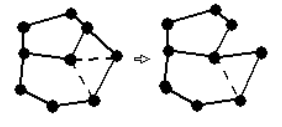

(b) Edge swap for vertex deletion

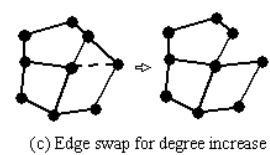

(c) Edge swap for degree increase
Figure 3. Graph simplification

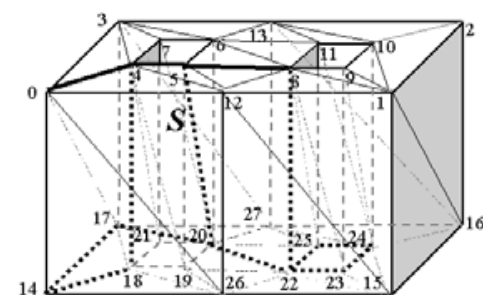

(a) Double Tonus Tessellated Surface

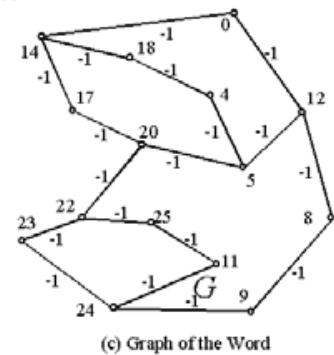

(c) Graph of the Word

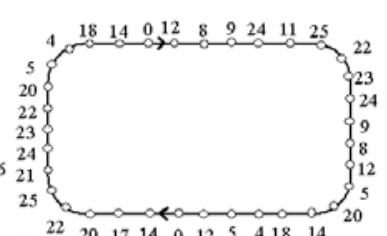

(b) A word representation of the surface

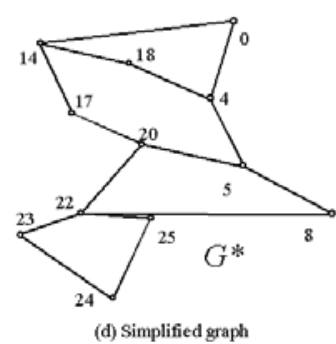

Figure 4: Graph construction and simplification for a double Torus
$(M S T)$ for $G$ and then adding the remaining edges, $R E$. We needed an algorithm which extracts the circuits in the order of 0 -order circuits, order -1 circuits and then order 1 circuits. Since standard methods of cycle decomposition does not guarantee such specific decomposition we adopted a special traversal strategy.

For extraction of 0 -order circuits, traversal is allowed only along 0 -order edges in MST. A 0 -order edge is picked up from $R E$ and added in MST to start the process of circuit construction from its two ends. If at any point a 0 -order edge is not available in the $M S T$ for traversal, we refer $R E$ and more 0 -order edges are picked up to complete the circuit. All but one of the 0 -order edges used is replaced back in $R E$ to prevent duplication of circuits extracted in subsequent stages. Used edges in $R E$ are marked in order to prevent initiation of another 0 -order circuit extraction using these edges. After all 0 -order circuits are extracted; each edge in $R E$ is used to extract a circuit in the usual manner and are classified based on the type of edges in the circuit.

\subsection{Surface Characterization}

Let, in $G, C_{0}, C_{1}, C_{2}$ be the number of circuits of orders $0,-1$ and 1 respectively. When $S$ is open, then union of $C_{0}, C_{1}, C_{2}$ may not give all the edges in $G$. The remaining edges in $G$ are then grouped together using simple linear traversal to construct paths. Edges in a path cannot be order-0; they are either all of order 1 or all of order -1. Let $L_{1}$ and $L_{2}$ be the number of paths of order -1 and 1 respectively. Using $C_{0}, C_{1}, C_{2}, L_{1}$ and $L_{2}$ surfaces can be classified as follows.

1. $C_{0}=0$ implies there is no boundary circle; hence the surface is closed and $L_{1}=L_{2}=0$; otherwise the surface is open.

2. $C_{2}=0$ and $L_{2}=0$ imply there is no order 1 edge in $G$; hence the surface is orientable

3. $C_{2} \neq 0$ or $L_{2} \neq 0$ imply the surface is non-orientable.

4. For orientable surfaces $\left(C_{2}=0\right.$ and $\left.L_{2}=0\right)$,

a. $C_{0}=0$ and $C_{1}=0$ imply the surface is homeomorphic to a sphere;

b. $C_{0} \neq 0$ and $C_{I}=0$ imply the surface is homeomorphic to a planar surface with $C_{0}$ boundary circles and $L_{1}=C_{0}-1$ substantial arcs; in particular, $C_{l}=1$ implies the surface is a disc;

c. $C_{1} \neq 0$ then $C_{1}=2 \mathrm{~g}$ where $\mathrm{g}$ is the genus of the surface; the surface is homeomorphic to a sphere with $C_{l} / 2=g$ handles, $C_{0}$ boundary circles and $L_{1}$ substantial arcs;

5. For non-orientable surfaces $\left(C_{2} \neq 0\right.$ or $\left.L_{2} \neq 0\right)$, since adding a handle is equivalent to adding two cross-caps to a sphere [13], we consider $k=C_{1}+C_{2}$; then

a. $\quad C_{0}=1$, and either $k+L_{2}=1$, or $L_{1}=1$, imply the surface is a Möbius strip.

b. $C_{0}=0$ and $k \neq 0$ imply the surface is self-intersecting, closed and homeomorphic to a sphere with $k$ cross-caps; in addition,

i. $k=1$ implies the surface is a map of the projective plane;

ii. $k=2$ implies the surface is a map of the Klein's bottle;

c. $\quad C_{0} \neq 0$ and $k \neq 0$ imply surface is open and homeomorphic to a sphere with $k$ cross-caps and $C_{0}$ boundary circles and $L_{1}+L_{2}$ substantial arcs;

In all the cases above, the actual geometric description of the substantial arcs/circles and boundary circles is obtained by mapping $C_{0}, C_{1}, C_{2}$ circuits and $L_{1} L_{2}$ paths on the surface. This, in turn, is easily done by using the coordinate of the points associated with the vertices in $G$. 


\section{ILLUSTRATIVE EXAMPLES}

The methodology described above has been implemented using $\mathrm{C}++$ and the graphical output is generated using OpenGL. The results are shown in a tabulated form for the sake of brevity in Table 1. The dark lines in the graphics indicate the detected substantial arcs/circles and the lighter lines indicate the detected boundary circles. Two boundary circles sharing one point are interpreted as part of a single circle. Input data file is in the format of indexed triangles: first coordinates of the vertices are specified followed by a list of triplets of vertex indices. In the examples shown, data for open surfaces are generated by omitting one or more triangles from the input data file. Program can handle any single component manifold surface: closed, open, orientable, and non-orientable.

Discussion: The program has been tested for all standard surfaces such as sphere, Möbius strip, torus and their various combinations obtained by surgery. It is interesting to observe that in example 7, the surface obtained from two Klein's bottles can result in a orientable surface, which is a self-intersecting embedding of a Torus. For a torus containing about 15000 triangles, it took about 8 seconds for word formation and 1.5 seconds for classification and graph simplification on a Pentium-4 PC. Work is underway to extend the method for handling non-manifold surfaces.

\section{Table 1 Illustrative Examples}

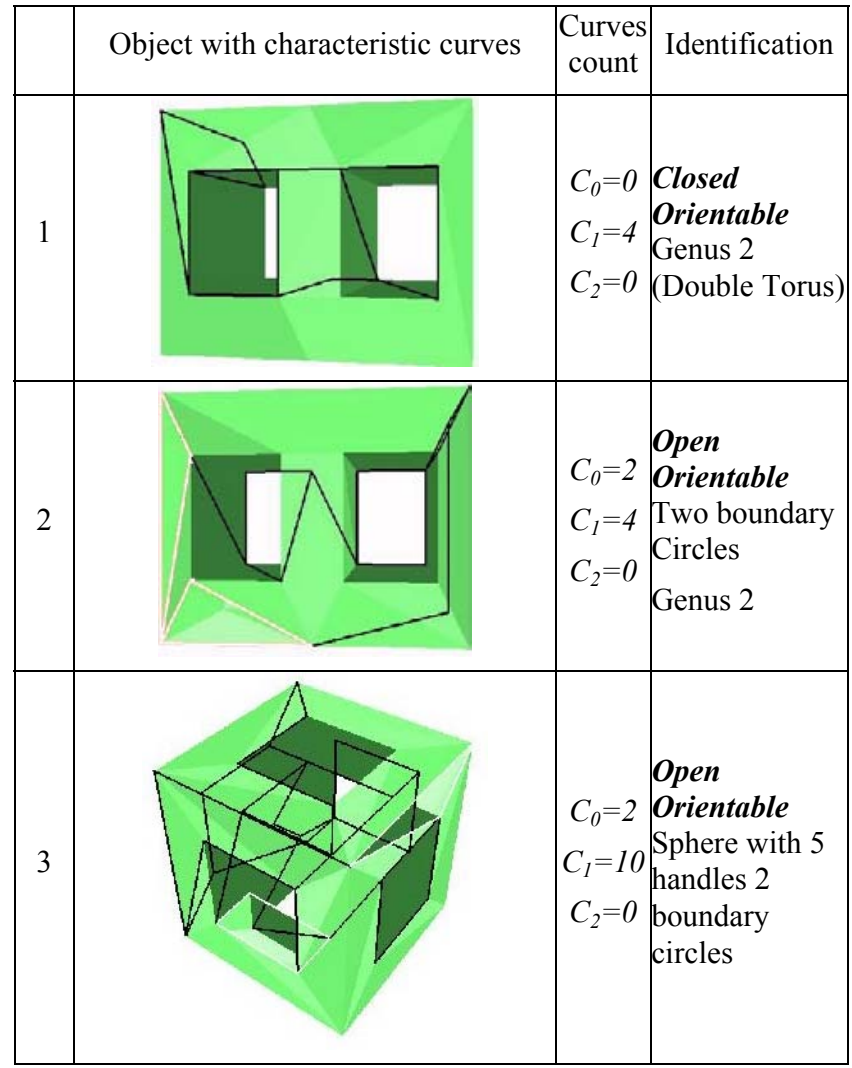

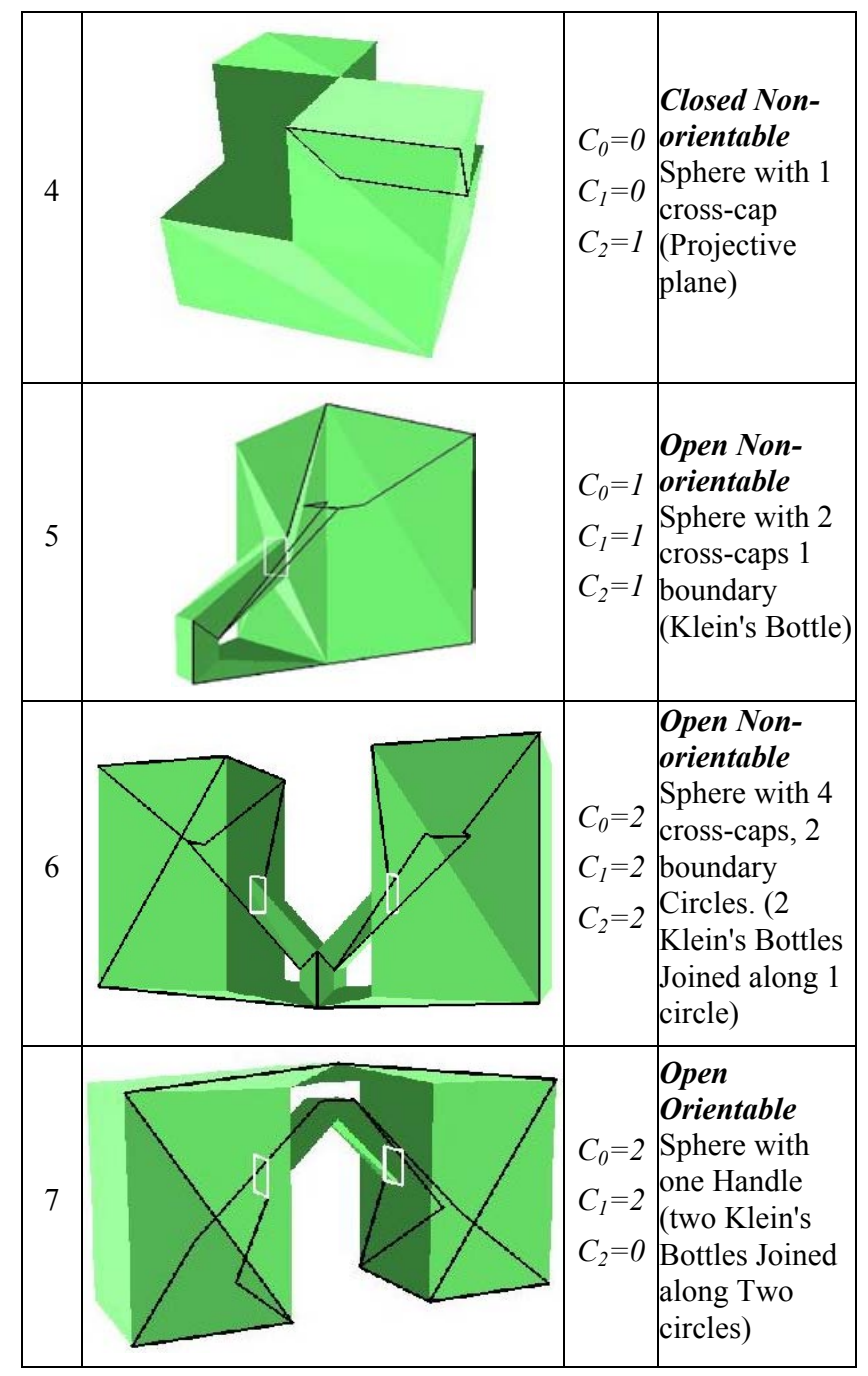

\section{CONCLUSIONS}

The paper presented a novel graph based methodology for topological analysis of any triangulated 2-manifold. The method constructs a graph out of the non-canonical word polygon obtained by sequential gluing of the triangles. The edges of the graph are classified according to their occurrence in the word and the circuits in the graph are classified based on the types of edges they contain. These circuits along with the paths that do not participate in the circuits are then used to characterize the surface. Since the edges in the word are only used for characterization, which is orders of magnitude less compared to the total number of triangle and edges in the surface, the procedure is efficient. Since the required graph decomposition can be done efficiently, the implementation is simple. Most importantly the validity of the method can be easily verified geometrically because it unifies the two traditional concepts of surface characterization based on canonical words and substantial arc/circles. The efficacy of the method has been demonstrated with a number of illustrative examples. 


\section{REFERENCES}

[1] Agarwal, S.C. and Waggenspack, W.N., Decomposition Method for Extracting Face Topologies from Wireframe models, Computer Aided Design, v24, 1992, pp. 123-140.

[2] Akleman, E. and Chen, J., Guaranteeing the 2-manifold Property for Meshes with Doubly Linked Face List, Int. Jl. Shape Modeling, v5 n2, 1999, pp.159-177.

[3] Bern, M. et al., Emerging Challenges in Computational Topology, Report of NSF Workshop on Computational Topology, June-1999.

[4] Biasotti, S., Topological Techniques for Shape Understanding, http://citeseer.nj.nec.com/biasotti01 topological.html.

[5] Carter, J.S., How Surfaces Intersects: An Introduction to Topology, 2nd ed. vol. 2, World Scientific Publications, 1995.

[6] Dey, T.K., Edelsbrunner and Guha, S., Computational Topology, In Adv. in Discr. and Comput. Geom. (Eds. Chazelle, B. et al.), Contemp. Math., v223, 1999, pp. 109143 ,

[7] Dey, T.K. and Guha, S., Computing Homology Groups of Simplical Complexes in R3, J. ACM, v45 n2, 1998, pp. 266287,

[8] Dey, T.K. and Schipper, H., A New Technique To Compute Polygonal Schema for 2-Manifolds with Application to NullHomotopy Detection, Discrete and Computational Geometry, v14, 1995, pp. 93-110.

[9] Dunlaing, C.O., Watt, C. and Wilkins, D., Homeomorphism of 2-Complexes is Equivalent to Graph Isomorphism, International Journal of Computational Geometry \& Applications, v10 n5, 2000, pp. 453-476.

[10] Erickson, J. and Har-Peled, S., Optimally Cutting a Surface into a Disk, Proc. 18th Annu., ACM Sympos. Comput. Geom., 2002, pp. 244-253.

[11] Fegurson, H., Rockwood, A. and Cox, J., Topological Deign of Sculptured Surfaces, In Edwin E. Catmull, editor, Computer Graphics (SIGGRAPH '92 Proceedings), v 26 n2, 1992, pp. 149-156.

[12] Fitzhorn, P.A., Language of Topologically Valid Bounding Manifolds, Computer Aided Design, Vol. 22, 1990, pp. 407416.

[13] Floater, M.S., Parameterization and Smooth Approximation of Surface Triangulations, Comp. Aided Geom. Design, v 14, 1997, pp. 231-250.

[14] Fomenko, A.T., Kunii, T. L., Topological Modeling for Visualization, Spinger-Verlag, Tokyo, 1997.

[15] Grzeszczuk, R.P., Hung, M., and Kauffman L.H., PhysicallyBased Stochastic Simplification of Mathematical Knots, IEEE Trans. on Visualization and Computer Graphics, 1997, pp. 262-272.

[16] Guo, B., Surface Reconstruction from Points to Splines, Computer Aided Design, v29 n4 1997, pp. 269-277.

[17] Hanrahan, P.M., Creating Volume Models from Edge-Vertex Graphs, ACM Computer Graphics, 1982, pp. 77-84.
[18] Harary, F., Graph Theory, Narosa Publishing House, New Delhi, 2000.

[19] Hart, J.C., Computational Topology for Shape Modeling, Proc. of Shape Modeling International '99, pp. AizuWakamatsu, Japan, March, 1999, pp. 36-45.

[20] Hart, J.C., Morse Theory for Implicit Surface Modeling, Mathematical Visualization, H-C Hege and K. Polthier, Eds., Springer-Verlag, Oct. 1998, pp. 257-268.

[21] Hart, J.C., Using the CW-Complex to Represent the Topological Structure of Implicit Surfaces and Solids, Proc. Implicit Surfaces '99, Eurographics/SIGGRAPH, Sept. 1999, pp. 107-112

[22] Hilaga, M., Shinagawa, Y., Kohmura T. and Kunii T. L., Topology Matching for Fully Automatic Similarity Estimation of 3D Shapes, SIGGRAPH, 2001, pp. 203-212

[23] Kartasheva, E., Reduction of h-Genus Polyhedrons Topology, Int. Jl. Shape Modeling, v5 n2, 1999, pp. 179-194.

[24] Lazarus, F. et al., Computing Canonical Polygonal Schema of an Orientable Triangulated Surface, Proc. $17^{\text {th }}$ Annu. ACM Symp. Comp. Geom., 2001, pp. 80-89.

[25] Leeuw, W. and Liere, R., Multi-Level Topology for Flow Visualization, Computer and Graphics, 2000, pp. 325-331.

[26] Ma, W. and Kruth, J.P., Parameterization of Randomly Measured Points for Least Squares Fitting of B-spline Curves and Surfaces, Computer Aided Design, v27 n9 1995, pp. 663-675.

[27] Ma, Y. and Luo, R.C., Topological Method for Loop Detection of Surface Intersection Problems, Computer Aided Design, 1995, pp. 811-820.

[28] Mantyla, M., An Introduction to Solid Modeling, Computer Science Press, 1976.

[29] Oblonsek, C. and Guid, N., A Fast Surface-Based Procedure for Object Reconstruction from 3D Scattered Points, Computer Vision and Image Understanding, v69 n2, 1998, pp. 185-195.

[30] Shawe-Taylor, J. and Pisanski, T., Homeomorphism of 2Complexes is Graph Isomorphism Complete, SIAM Journal on Computing, v 23 n1, 1994, pp. 120-132.

[31] Sinsgswa, Y., Kunii, T.L. and Kergosies Y.L. Surface Coding Based on Morse Theory, IEEE Computer Graphics, September-1991, pp. 66-78.

[32] Stander, B.T. and Hart, J.C., Guaranteeing the Topology of an Implicit Surface Polygonalization for Interactive Modeling, Proc. SIGGRAPH 97, Aug. 1997, pp. 279-286.

[33] Steiner, D. and Fischer, A., Cutting 3D Freeform Objects with Genus-n into Single Boundary Surfaces using Topological Graphs, Proc. 7th ACM Symp. Solid Modeling and Applications, Saarbrücken, Germany, 2002, pp. 336-343.

[34] Vegter, G. and Yap, C.K., Computational Complexity of Combinatorial Surfaces, Proc. 6th ACM Symp. On Computational Geometry, 1990, pp. 102-11. 